\title{
Encaminhamentos da atenção primária para avaliação neurológica em Porto Alegre, Brasil
}

\author{
I 'Alessandro Finkelsztejn, 'Lisiane Morelia W. Acosta, ${ }^{3}$ Rafael do Amaral Cristovam, \\ ${ }^{4}$ Gisele Silva de Moraes, ${ }^{5}$ Michele Kreuz, ${ }^{6}$ Anne Orgler Sordi, ${ }^{7}$ Pablo Cambeses Souza, \\ 8Tatiana Falcão Eyff, ${ }^{9}$ Anelise Martins Fracasso, ${ }^{10}$ Márcia Lorena F. Chaves I
}

Resumo: No Sistema Único de Saúde do Brasil (SUS), os níveis de atenção à saúde se inserem no modelo hierárquico através do sistema de referência e contrarreferência. Em um projeto para atender às demandas represadas do nível primário ao secundário, denominado "Mutirão da Saúde", realizado em Porto Alegre, os médicos neurologistas do Hospital de Clínicas de Porto Alegre, após cada atendimento realizado, responderam a um instrumento para avaliar os encaminhamentos realizados pela rede de atenção primária à saúde (APS). A avaliação foi positiva, mostrando que os encaminhamentos foram realmente necessários em 85\% dos casos; entretanto, foi parcialmente contraditória, tendo em vista que $41,7 \%$ dos encaminhamentos eram situações clínicas que deveriam ser manejadas no atendimento primário. A avaliação também revelou que $50 \%$ dos casos necessitavam de exames complementares. Devido a uma possível regionalização aleatória dos encaminhamentos, o resultado não nos possibilitou uma estimativa apropriada da prevalência por territórios das unidades de APS, informação esta importante para a organização do fluxo de encaminhamentos e planejamento dos recursos alocados pelos gestores, tanto locais quanto municipais.

\author{
${ }^{1}$ Médico neurologista, médico \\ concursado do Hospital de \\ Clínicas de Porto Alegre; \\ mestre em Epidemiologia pela \\ Universidade Federal do Rio \\ Grande do Sul (UFRGS). \\ Endereço eletrônico: \\ afinkels@portoweb.com.br. \\ 2 Enfermeira com \\ especialização em \\ Enfermagem em Saúde \\ Pública. Endereço eletrônico: \\ lisacosta@uol.com.br. \\ ${ }^{3}$ Acadêmico de Medicina da \\ UFRGS. Endereço eletrônico: \\ rafael.cristovam@ufrgs.br. \\ ${ }^{4}$ Acadêmica de Medicina da \\ UFRGS. Endereço eletrônico: \\ Gisele.moraes@ufrgs.br. \\ ${ }^{5}$ Médica. Endereço eletrônico: \\ mkreuz@hcpa.ufrgs.br. \\ ${ }^{6}$ Médica. Endereço eletrônico: \\ annesordi@yahoo.com.br. \\ ${ }^{7}$ Acadêmico de Medicina da \\ UFRGS. Endereço eletrônico: \\ pablocambeses@gmail.com. \\ ${ }^{8}$ Acadêmica de Medicina da \\ UFRGS. Endereço eletrônico: \\ tatieyff@terra.com.br. \\ ${ }^{9}$ Médica. Endereço eletrônico: \\ anemf@hotmail.com. \\ ${ }^{10}$ Professora da UFRGS; chefe \\ do Serviço de Neurologia do \\ Hospital de Clínicas de Porto \\ Alegre. Endereço eletrônico: \\ mchaves@hcpa.ufrgs.br.
}




\section{Introdução}

A Constituição brasileira promulgada em outubro de 1988 estabeleceu, em seu artigo 196, que "A saúde é um direito de todos e um dever do Estado, garantida mediante políticas sociais e econômicas que visem à redução do risco de doença e de outros agravos e ao acesso universal igualitário às ações e serviços para sua promoção, proteção e recuperação" (BRASIL, 1988). Este marco histórico na transformação do modelo de saúde vigente no país foi regulamentado pela Lei ${ }^{\circ}$ 8.080, de 19 de setembro de 1990, estabelecendo que a direção do SUS seja única, exercida no âmbito nacional pelo Ministério da Saúde; no âmbito estadual, pela Secretaria Estadual de Saúde; e nos municípios, pela Secretaria Municipal de Saúde (BRASIL, 1990).

Além de um nível hierárquico de gestão descentralizada, o SUS estabelece níveis de complexidade da assistência, denominados como primário, secundário e terciário. Na cidade de Porto Alegre-RS, a atenção primária à saúde (APS) do SUS é realizada pelas equipes de saúde da família (ESF) e unidades básicas de saúde tradicionais (UBS).

Os casos que necessitam de avaliação especializada e possível realização de exames complementares com tecnologia de alto custo são encaminhados para os ambulatórios especializados do nível secundário e hospitais terciários, entre os quais se encontra o Hospital de Clínicas de Porto Alegre (HCPA), um hospital universitário vinculado à Universidade Federal do Rio Grande do Sul, local da realização deste estudo. O sistema de encaminhamentos, chamado de "Referência e Contrarreferência" em nosso meio, faz parte de um dos atributos essenciais da APS, a coordenação única da atenção à saúde. Esta coordenação prevê um cuidado compartilhado entre o médico generalista e o especialista, de maneira a integrar as informaçōes dos cuidados entre os diversos níveis do sistema de saúde.

Em Porto Alegre, esse modelo funciona através de um formulário chamado "Ficha de Referência e Contrarreferência", na qual o médico da assistência primária preenche os campos com os dados do caso. Ao reencaminhar ao posto de origem, o médico de referência informa o diagnóstico e sugere a conduta a ser realizada no nível primário. Caso haja maior complexidade no caso, o paciente passa a ser atendido conjuntamente no serviço especializado (STARFIELD, 1994). Entretanto, verifica-se uma demanda reprimida de consultas na atenção secundária e terciária, em especial na Neurologia, onde se atingiu um tempo de espera de até quatro 
anos, em levantamento feito no ano de 2006 em Porto Alegre. Com o intuito de aumentar a disponibilidade de atendimento nas especialidades, a Secretaria Municipal de Saúde traçou um projeto denominado "Mutirão da Saúde 2006", que visou a promover o atendimento de toda esta demanda reprimida de consultas (60.000 atendimentos) num curto espaço de tempo, abrangendo todas as especialidades, inclusive a Neurologia.

Há uma ideia corrente de que os pacientes com queixas neurológicas são mal encaminhados para o atendimento especializado. Nosso estudo tem a finalidade principal determinar se realmente isto ocorre na Saúde Pública em Porto Alegre.

O Serviço de Neurologia do HCPA participou desse projeto durante três meses (agosto, setembro e outubro de 2006), tendo oferecido mil atendimentos, porém realizando apenas 439 consultas. Buscando avaliar os encaminhamentos realizados pela atenção primária, este estudo se propôs a determinar o perfil dos pacientes encaminhados ao Serviço de Neurologia do HCPA, as queixas principais, os motivos dos encaminhamentos, hipóteses diagnósticas mais comuns e a proporção de pacientes com possibilidade de terem seus problemas resolvidos na APS segundo avaliação dos especialistas, e compará-los com os dados existentes na literatura. Este estudo tem caráter inovador e factível, sendo relevante ao planejamento e gestão da saúde no nível local e nacional, pois há poucos estudos semelhantes na literatura.

\section{Métodos}

Este foi um estudo transversal, realizado no Serviço de Neurologia do HCPA, no período de agosto a outubro de 2006. A população estudada foi de 439 pacientes referidos pelos serviços de APS do município através do "Mutirão da Saúde", constituindo uma amostra de conveniência. $\mathrm{O}$ instrumento de pesquisa foi aplicado pelos pesquisadores aos médicos neurologistas no término de cada consulta, após obtenção do termo de consentimento livre e esclarecido. Ao término do "Mutirão da Saúde", todos os dados foram analisados de acordo com os objetivos propostos.

$\mathrm{O}$ instrumento de pesquisa foi baseado no modelo da ficha de referência e contrarreferência, sendo realizada análise descritiva dos casos através da média, desvio-padrão, frequência e percentual.

Este estudo foi aprovado pelo Comitê de Ética em Pesquisa do HCPA (CEP/ CONEP) e foi conduzido dentro da legislação brasileira, segundo a Resolução no 196/96 do Conselho Nacional de Saúde e dos princípios da Declaração de Helsinque. 


\section{Resultados}

O perfil da população do estudo, em relação à faixa etária e sexo, foi de 59\% do sexo feminino, sendo a idade mínima, em ambos os sexos, de um ano e a máxima, no sexo feminino de 87 anos e 81 anos no sexo masculino. Observa-se que, dentre os homens, há menor quantidade de consultas na faixa de 20-40 anos.

Figura 1. Distribuição de idade entre o sexo masculino

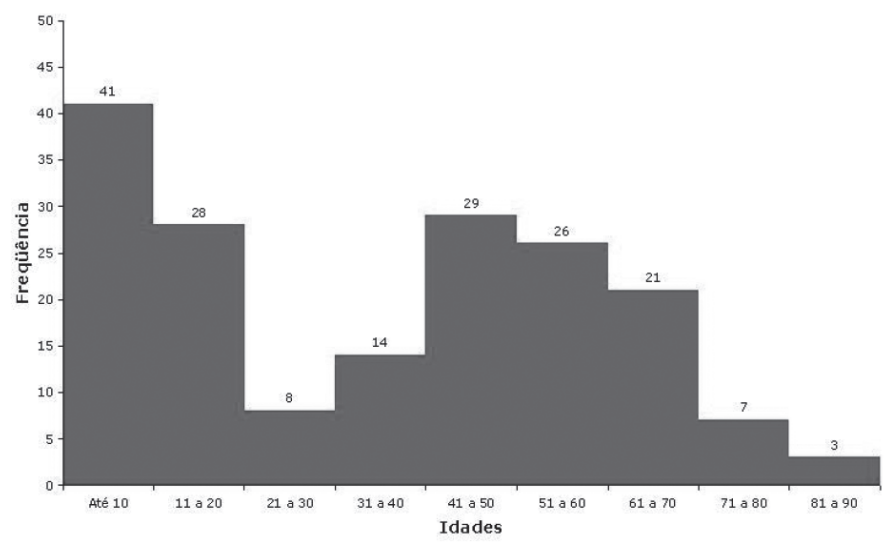

* Idade expressa em anos

* Frequência expressa em números absolutos

Figura 2. Distribuição da idade entre o sexo feminino

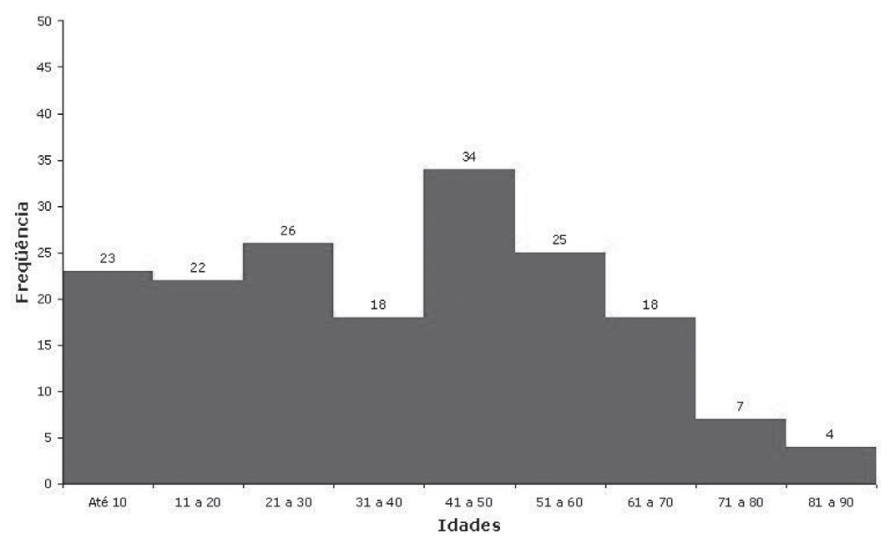

* Idade expressa em anos

* Frequência expressa em números absolutos 
Embora os sintomas mais prevalentes em ambos os sexos tenham sido cefaleia e convulsão, os demais sintomas diferem em magnitude entre os sexos. A queixa de alteração de comportamento se destaca no sexo masculino, principalmente em menores de 15 anos.

Figura 3. Distribuiç̧ão dos sintomas mais prevalentes

\begin{tabular}{|l|c|c|}
\hline Sintoma & $\begin{array}{c}\text { Freqüência } \\
\text { absoluta }\end{array}$ & $\begin{array}{c}\text { Freqüência } \\
\text { relativa (\%) }\end{array}$ \\
\hline Cefaléia & 143 & 32,9 \\
\hline Convulsões & 59 & 13,6 \\
\hline $\begin{array}{l}\text { Alteração no } \\
\text { comportamento }\end{array}$ & 38 & 8,8 \\
\hline Diminuição da memória & 27 & 6,2 \\
\hline Deficit de aprendizado & 18 & 4,1 \\
\hline Perda da consciência & 17 & 3,9 \\
\hline Diminuição da & 14 & 3,2 \\
\hline sensibilidade & 13 & 3,0 \\
\hline Tonturaşlertigens & 9 & 2,1 \\
\hline Distúrbio do sono & 9 & 2,1 \\
\hline Dor neuropática & 87 & 20,0 \\
\hline Outros & 434 & 100 \\
\hline Total & & \\
\hline
\end{tabular}

Percebe-se que a distribuição da necessidade de exames complementares solicitados pelo médico neurologista não teve diferença em relação ao sexo e se manteve em torno dos $50 \%$. 
Figura 4. Necessidade de exames complementares

\begin{tabular}{|l|c|c|c|}
\hline & \multicolumn{2}{|l|}{ Sexo } & \\
\hline Exame Complementar & $\begin{array}{c}\text { Masculino } \\
\mathbf{( \% )}\end{array}$ & $\begin{array}{c}\text { Feminino } \\
\mathbf{( \% )}\end{array}$ & Total (\%) \\
\hline Sim & $99(55,6)$ & $133(51,6)$ & $232(53,2)$ \\
\hline Nã̃o & $79(44,4)$ & $125(48,4)$ & $204(46,8)$ \\
\hline
\end{tabular}

A avaliação do especialista, considerando-se em torno de $85 \%$ o encaminhamento do nível primário como necessário, não diferiu entre sexos e aparentemente mostrou uma boa qualidade do encaminhamento, sob a ótica do neurologista.

Figura 5. Necessidade de ter sido feito o encaminhamento

\begin{tabular}{|l|l|l|l|}
\hline & Sexo & \\
\hline $\begin{array}{l}\text { Encaminhamento } \\
\text { necessário }\end{array}$ & $\begin{array}{l}\text { Masculino } \\
\text { (\%) }\end{array}$ & $\begin{array}{l}\text { Feminino } \\
\mathbf{( \% )}\end{array}$ & $\begin{array}{l}\text { Total } \\
\text { (\%) }\end{array}$ \\
\hline Sim & $156(87,6)$ & $221(85,7)$ & $377(86,5)$ \\
\hline Nẵo & $22(12,4)$ & $37(14,3)$ & $59(13,5)$ \\
\hline
\end{tabular}

A distribuição das hipóteses diagnósticas é apresentada na tabela abaixo, apresentando variado grau de complexidade. 
Figura 6. Hipóteses diagnósticas encontradas

\begin{tabular}{|l|l|l|}
\hline Hipótese & $\begin{array}{l}\text { Freqüência } \\
\text { absoluta }\end{array}$ & $\begin{array}{l}\text { Freqüência } \\
\text { relativa (\%) }\end{array}$ \\
\hline Cefaléia Benigna & 122 & 28,2 \\
\hline Epilepsia & 62 & 14,4 \\
\hline TDAH & 30 & 6,9 \\
\hline AVC & 18 & 4,2 \\
\hline RDNP & 18 & 4,2 \\
\hline Sincope & 18 & 4,2 \\
\hline Demência & 18 & 4,2 \\
\hline Depressåo & 16 & 3,7 \\
\hline Neuropatia & 15 & 3,5 \\
\hline Hérnia discal & 14 & 3,2 \\
\hline Tumores & 12 & 2,8 \\
\hline Hipertensåo Intracraniana & 9 & 2,1 \\
\hline Distúrbio do sono & 7. & 1,6 \\
\hline Parkinson & 6 & 1,4 \\
\hline Jertigemllabiintite & 6 & 1,4 \\
\hline Distúrbio psiquiátrico & 5 & 1,2 \\
\hline Distúrbio do Movimento & 3 & 0,7 \\
\hline Eibromialgia & 1 & 0,2 \\
\hline Alcoolismo & 1 & 0,2 \\
\hline Outros & 51 & 11,8 \\
\hline Total & 432 & 100 \\
\hline & & \\
\hline
\end{tabular}

Em virtude de uma regionalização dos casos referidos às diferentes unidades de atenção secundária, não foi possível determinar a prevalência dos problemas neurológicos por região, tendo sido apenas distribuído o número de casos encaminhados ao HCPA por unidade de atenção primária de origem. Avaliandose as cinco unidades com maior número de casos encaminhados, a necessidade deste encaminhamento se manteve entre 80 e $100 \%$, segundo os especialistas. 


\section{Discussão}

Este estudo possui algumas limitações, como o baixo número absoluto de atendimentos, o elevado número de faltas às consultas agendadas, o fato de a amostra ter sido de conveniência, o atendimento de pacientes em outros hospitais e o desconhecimento dos critérios para escolha de um ou outro hospital.

Os principais pontos fortes do estudo foram: o pioneirismo deste tipo de avaliação de encaminhamentos para avaliação neurológica em nosso estado, levando a um melhor conhecimento da distribuição das queixas neurológicas na população, e a possibilidade de esses dados contribuírem para um melhor planejamento do ensino da Neurologia.

A comparação das frequências das queixas neurológicas em nossa amostra com as apresentadas em outros estudos revela que a distribuição das queixas neurológicas assemelha-se mais ao estudo de Nitrini (NITRINI; FERRI-DE-BARROS, 1996), talvez devido ao fato de a amostra ter sido extraída de encaminhamentos ambulatoriais. Comparando a prevalência das queixas neurológicas em nossa amostra, verificamos importantes diferenças, sobretudo ao compararmos com os estudos de Osuntokun (OSUNTOKUN et al., 1987), Bittencourt (TREVISOLBITTENCOURT et al., 2001) e Ferri-de-Barros (FERRI-DE-BARROS et al., 2000). Osuntokun e colaboradores apresentaram dados de prevalência na população nigeriana (estudo de base populacional). Comparando com o estudo de Bittencourt e e colaboradores (TREVISOL-BITTENCOURT et al., 2001), encontramos muitas diferenças na prevalência das queixas, visto ter sido utilizada uma amostra de pacientes em perícia médica. $\mathrm{O}$ estudo de Ferri-de-Barros et al. utiliza amostra de pacientes que procuraram o pronto-socorro de três hospitais públicos. Tendo em vista essas diferenças, reforça-se o conceito do nosso estudo como um modelo de avaliação dos encaminhamentos praticados pela atenção primária em nossa cidade, podendo vir a ser utilizado em outras especialidades.

Tendo em vista uma percepção corrente (não baseada em evidências) de que muitos encaminhamentos ao neurologista poderiam ser resolvidos na própria APS, através de melhor capacitação dos profissionais de saúde para a abordagem dos problemas mais prevalentes, este estudo mostrou uma realidade diferente dessa suposição, de acordo com os médicos especialistas em neurologia do HCPA. Os encaminhamentos da APS foram necessários num percentual superior a $85 \%$, o que demonstra adequado grau de compreensão da gravidade dos casos por parte dos médicos do nível primário. 
Analisando-se essa taxa por posto de saúde, verificou-se que a mesma se manteve estável, inclusive entre as cinco unidades com maior numero de casos. Contudo, ao analisarmos com mais cautela os dados, principalmente os referentes aos diagnósticos encontrados, 41,7\% destes se referem a patologias ou situações que usualmente não precisariam ter sido encaminhados ao especialista (cefaleias benignas, AVC nãoagudo, depressão, distúrbio psiquiátrico, fibromialgia). Por exemplo, cefaleias benignas e outras situações podem e devem ser diagnosticadas com segurança pelo médico da atenção primária. Por isso, verificamos com esta análise mais aprofundada, que, considerando os diagnósticos levantados, há uma incoerência de julgamento pelos próprios neurologistas, pois enquanto estes consideravam que mais de $85 \%$ dos casos deveriam ter sido encaminhados para sua avaliação, também diagnosticaram $41,7 \%$ dos casos de acordo com diagnósticos que - senso comum - não precisariam ter sido encaminhados para avaliação com neurologista.

As causas dessa incoerência podem ser várias, mas três devem ser mencionadas por serem as mais prováveis: sentimentalismo ou compaixão pelo paciente, insegurança ou falta de autoconfiança e a reserva de mercado. Não há como provarmos a existência destas causas, mas devemos apontá-las e discuti-las no nosso próprio serviço e entre os neurologistas de nossa cidade.

As diferenças observadas entre faixa etária e sexo levantam algumas hipóteses: a razão de a mulher apresentar mais problemas neurológicos ocorre por ser ela mais cuidadosa com sua saúde, frequentar com mais periodicidade os postos de saúde, e também por ser ela quem identifica o problema nos filhos, fazendo ter, tanto no sexo masculino como no feminino, um percentual semelhante em menores de 15 anos. Percebe-se claramente a queda de casos no histograma do sexo masculino entre a faixa etária de 20 a 40 anos, na qual o homem está mais independente do cuidado feminino. Ressaltamos também que o período de funcionamento dos postos, geralmente em horário comercial, não propicia acesso adequado para os trabalhadores de uma maneira geral, sobretudo os do sexo masculino. Distorções como estas são desafios constantes para gestores que buscam qualificar as "portas de entrada" do sistema de saúde, sendo importante a adoção de horários alternativos para a inclusão desta população.

Em relação à distribuição geográfica dos casos, é provável que tenha ocorrido uma regionalização dos encaminhamentos, pois outros serviços de neurologia do município também estiveram envolvidos no atendimento. Portanto, esta análise 
se mostra inapropriada para estimar a prevalência de agravos neurológicos, informação que acreditamos ser importante para a organização do fluxo de encaminhamentos e planejamento dos recursos alocados pelos gestores, tanto municipal quanto os distritais, na busca da otimização dos gastos públicos em saúde e da resolução das listas de espera, que permanecem como importante problema em todos os níveis de atenção (KURTZKE; HOUFF, 1995).

\section{Referências}

BRASIL. Senado Federal. Constituição da República Federativa do Brasil. Brasília: Senado Federal, 1988.

BRASIL. Ministério da Saúde. Lei no 8.080. Dispõe sobre as condições para a promoção, proteção e recuperação da Saúde, a organização e financiamento dos serviços correspondentes e dá outras providências. Diário Oficial da Uniāo, Brasília, 31 dez. 1990.

FERRI-DE-BARROS, J. E. et al. Transtornos neurológicos mais frequentes: contribuição para a definição de temas do conteúdo programático do curso de neurologia para a graduação médica. Arq Neuropsiquiatr, v. 58, n. 1, p. 128-135, 2000.

KURTZKE, J. F.; HOUFF, S. A. A primary care plan for neurology. Neurology, v. 45, p. $1.052-$ $1.061,1995$.

NITRINI, R.; FERRI-DE-BARROS, J. E. Que pacientes atende um neurologista? Alicerce de um currículo em neurologia. Arq Neuropsiquiatr, v. 54, n. 4, p. 637-644, 1996.

OSUNTOKUN, B. O. et.al. Neurological disorders in Nigerian africans: a community-based study. Acta Neurol Scand, n. 75, p. 13-21, 1987.

STARFIELD, B. Is primary care essential? Lancet, n. 344, p. 1.129-1.133, 1994.

TREVISOL-BITTENCOURT, P. C. et al. Condições mais frequentes em um ambulatório de perícia neurológica. Arq Neuropsiquiatr, v. 59, n. 2-A, p. 214-218, 2001. 


\section{Abstract}

Referrals from primary care to neurological assessment in the city of Porto Alegre, Brazil

In the Unified Health System in Brazil (SUS), the levels of health care fall within the hierarchical model with the reference and counter-reference systems. In a project to meet the repressed demands of primary and secondary levels, called "Mutirão da Saúde", held in Porto Alegre, the neurologists of the Hospital de Clinicas de Porto Alegre, after each call made, answered a questionnaire to assess referrals performed by the network of primary health care (PHC). The evaluation was positive, showing that the referrals were really needed in $85 \%$ of cases; however, it was partially contradictory, given that $41.7 \%$ of referrals were medical conditions that should be managed in primary care. The evaluation also revealed that $50 \%$ of cases needed further investigation. Due to a possible randomized regionalization of referrals, the result did not allow us to estimate the appropriate prevalence according to areas of the PHC units, information that is important to organize the flow of referrals and planning of resources allocated by managers, both local and municipal.

> Key words: health centers; public health; primary health care; neurologic manifestations. 